Neurosurg Focus 13 (4):Article 4, 2002 (Previously J Neurosurg 97:21-32, 2002) Click here to return to table of contents

\title{
Utility of preoperative functional magnetic resonance imaging for identifying language cortices in patients with vascular malformations
}

\author{
Nader Pouratian, Ph.D., Susan Y. Bookheimer, Ph.D., David E. Rex, B.S., \\ Neil A. Martin, M.D., And Arthur W. Toga, Ph.D. \\ Department of Neurology, Laboratory of Neuro Imaging; UCLA Brain Mapping Center, Department \\ of Psychiatry and Biobehavioral Sciences; Neuropsychiatric Institute and Hospital; Division of \\ Neurosurgery, UCLA School of Medicine, University of California, Los Angeles, California
}

\begin{abstract}
Object. The goal of this study was to evaluate the utility of preoperative functional magnetic resonance (fMR) imaging in the prediction of whether a given cortical area would be deemed essential for language processing by electrocortical stimulation mapping (ESM).

Methods. The authors studied patients with vascular malformations, specifically arteriovenous malformations (AVMs) and cavernous angiomas, in whom blood-flow patterns are not normal and in whom a perfusion-dependent mapping signal may be questionable. Ten patients were studied (seven harboring AVMs and three with cavernous angiomas). The authors used a battery of linguistic tasks, including visual object naming, word generation, auditory responsive naming, visual responsive naming, and sentence comprehension, to identify brain regions that were consistently activated across expression and comprehension linguistic tasks. In a comparison of ESM and fMR imaging activations, the authors varied the matching criteria (overlapping activations, adjacent activations, and deep activations) and the radii of influence of ESM $(2.5,5$, and $10 \mathrm{~mm})$ to determine the effects of these factors on the sensitivity and specificity of fMR imaging. The sensitivity and specificity of fMR imaging were dependent on the task, lobe, and matching criterion. For the population studied, the sensitivity and specificity of fMR imaging activations during expressive linguistic tasks were found to be up to 100 and $66.7 \%$, respectively, in the frontal lobe, and during comprehension linguistic tasks up to 96.2 and $69.8 \%$, respectively, in the temporal and parietal lobes. The sensitivity and specificity of each disease population (patients with AVMs and those with cavernous angiomas) and of individuals were consistent with those values reported for the entire population studied.

Conclusions. The authors conclude that preoperative fMR imaging is a highly sensitive preoperative planning tool for the identification of which cortical areas are essential for language and that this imaging modality may play a future role in presurgical planning for patients with vascular malformations.
\end{abstract}

KEY WORDS • brain mapping • functional magnetic resonance imaging • language cortex • arteriovenous malformation • electrocortical stimulation mapping

Neurosurgical resection of cerebral diseases, such as neoplasms and AVMs, near eloquent cortices can result in iatrogenic damage, such as paralysis and language deficits. The proximity of AVMs to eloquent cortices is, consequently, one of the three major factors used to predict surgical risk. ${ }^{34}$ To maximize resection of pathological tissue, spare eloquent cortices, and reduce surgical risk, several functional mapping procedures have been developed, including intraoperative ESM,${ }^{26}$ preoperative fMR imaging, ${ }^{11}$ and intraoperative optical imaging of intrinsic signals. ${ }^{14}$ Electrocortical stimulation mapping is currently the gold standard of intraoperative functional cortical mapping because it has been shown to be a good predictor of the effects

Abbreviations used in this paper: $\mathrm{AVM}=$ arteriovenous malformation; $\mathrm{BOLD}=$ blood oxygen level-dependent; $\mathrm{EPI}=$ echoplanar imaging; $\mathrm{ESM}=$ electrocortical stimulation mapping; $\mathrm{fMR}=$ functional magnetic resonance; PET = positron emission tomography. of resection. ${ }^{12}$ The usefulness of ESM, however, is limited by relatively low spatial resolution and long (approximately 1 hour) mapping times. Preoperative fMR imaging offers higher spatial resolution, the ability to map multiple tasks before surgery, and the potential to reduce the time needed for intraoperative mapping. The validity of preoperative fMR imaging for surgical planning has yet to be fully validated, however, especially in patients in whom there are vascular malformations.

\section{Electrocortical Stimulation Mapping}

Electrocortical stimulation mapping is used to map the brain by applying currents directly to the surface of the brain intraoperatively, while the patient performs a task. Direct cortical stimulation induces a local temporary lesion that disrupts the normal functioning of that area of cortex. ${ }^{25}$ If stimulation of a certain cortical area is found to disrupt the patient's ability to perform a language task, it is consid- 
ered "essential" for language function. This form of mapping has been useful for predicting functional recovery; full recovery of language skills can be expected when the resection is no closer than $1 \mathrm{~cm}$ from essential language sites. ${ }^{12}$ Furthermore, ESM has been shown to be task specific. For example, in bilingual individuals, stimulation of some cortical areas may disrupt processing of only one language. ${ }^{27,28}$ Nevertheless, ESM has potential drawbacks, the most important of which are its potential to induce afterdischarge activity, the relatively long time required for mapping, and its limited spatial resolution.

\section{Functional MR Imaging}

Blood oxygen level-dependent fMR imaging is a functional neuroimaging method that maps the brain by detecting perfusion-related changes that are coupled to neuronal activity ${ }^{24}$ Several studies have demonstrated robust and reliable task-associated fMR imaging activations in language areas (in both frontal and perisylvian language sites). ${ }^{8,10,11}$ Nevertheless, the reliability of fMR imaging signals, which are based on perfusion, is unclear in patient populations in which blood-flow abnormalities exist (that is, in patients harboring AVMs or cavernous angiomas).

\section{Arteriovenous Malformations}

Brain AVMs are congenital lesions characterized by abnormal direct arteriovenous communications without an intervening capillary bed. The abnormal and turbulent flow associated with AVMs is normally marked by high-flow voids on structural MR images due to local disruption of magnetic fields. Because of the local disruptions in magnetic fields and abnormal blood-flow patterns, it is necessary to investigate further whether accurate and useful fMR imaging maps can be obtained in this patient population. Although fMR imaging activations have been identified in patients harboring AVMs,${ }^{1,2,16,22,32}$ no one has determined the correlation of these activations with intraoperative electrocortical stimulation maps.

In the most thorough study to date of the correlation of ESM and fMR imaging in neurosurgical patients, multiple language tasks were used and "activation from only one of the tasks [was] required to touch the language tag" for a positive match to be identified. ${ }^{11}$ FitzGerald and associates, ${ }^{11}$ who did not specifically study patients with vascular malformations, reported a very high sensitivity (percentage of positive ESM sites that colocalized with fMR imaging activations [range 81-92\%]), but simultaneously reported a very low specificity $(0-53 \%)$, meaning that many, if not all, ESM sites that proved negative for language also overlapped fMR imaging activations. Other attempts to correlate fMR imaging with ESM have been limited and include only a small number of study participants (1-3). ${ }^{18,23,31}$ More studies are therefore required to demonstrate the usefulness of fMR imaging to predict essential language sites.

The purpose of this study is to characterize the relationship between preoperative fMR imaging language maps with intraoperative ESM language maps in patients with vascular malformations. We compared intraoperative ESM with preoperative fMR imaging by using a combination of linguistic tasks to determine the specificity and sensitivity of this imaging modality relative to ESM. We also determined whether the predictive value of preoperative fMR imaging varies across different cortical areas and different disease populations.

\section{Clinical Material and Methods}

Ten patients (six men and four women; seven patients harboring AVMs and three harboring cavernous angiomas) were studied in accordance with University of California at Los Angeles Human Subjects Protection Committee guidelines. Informed consent was obtained from all patients before they underwent surgery. All patients were right handed. Left-hemisphere language localization was confirmed in all patients by a preponderance of fMR imaging activations in the left hemisphere, ${ }^{30}$ by ESM, and, in three patients, by superselective Wada testing. The AVMs were located either in the frontal lobe (precentral gyrus [two cases]) or the temporoparietal lobe region (within the sylvian fissure [five cases]) and ranged in size from $4 \mathrm{~mm}$ to $3 \mathrm{~cm}$.

\section{Preoperative fMR Imaging}

Preoperative fMR imaging was performed approximately 1 week before surgery. Magnetic resonance images were collected using a 3-tesla magnet (General Electric Medical Systems [Milwaukee, WI] equipped with EPI from Advanced NMR [Wilmington, MA]). Before functional images were obtained, a fast spin-echo sequence was used to generate high-resolution structural maps of each patient's brain (TR $5000 \mathrm{msec}$, TE $18 \mathrm{msec}$, flip angle $90^{\circ}$, matrix size $256 \times 256$, field of view $24 \times 18 \mathrm{~cm}, 363$-mm-thick axial slices with no gap). Multiple images were obtained in each patient to determine language function. For each functional trial, we used an EPI gradient-echo sequence (TR $2500 \mathrm{msec}$, TE $45 \mathrm{msec}$, matrix size $64 \times 64$; field of view $20 \mathrm{~cm}$ ) to collect 84 functional images over 17 axial slices (4-mm-thick slices with a 1-mm gap). Each functional image included three 30-second blocks of the same language task, with 30 seconds of rest between each activation block, starting and ending with a rest period. All language tasks performed in this study have been validated in healthy volunteers by using PET and fMR imaging. Language tests included visual object naming, ${ }^{5}$ word list generation, ${ }^{9}$ auditory responsive naming, ${ }^{4}$ visual responsive naming, and sentence comprehension ${ }^{10}$ (see later descriptions of these tests). Visual stimuli were presented through MR-compatible goggles, which were fitted over the patients' eyes before the patients were placed in the imaging unit. Binaural auditory stimuli were delivered via MR-compatible headphones. Both auditory and visual stimuli were controlled and presented using a Macintosh computer running MacStim software (Apple Computers, Inc., Cupertino, CA).

Before we began functional analysis, we first realigned images within a functional series with automated image registration by using rigid body transformations to correct for head motion. ${ }^{35}$ Functional activity was estimated by correlating the time course of the voxels in each functional image with a reference function. The reference function was a box-car reference function convolved with a model of the hemodynamic response by using software developed at our center. ${ }^{7}$ In some instances, fMR imaging trials were excluded because there had been excessive head motion during imaging, which was evident by large-magnitude activations at the edges of the brain and at interfaces of gray and white matter. 


\section{Preoperative fMR imaging of language in patients with AVMs}

To create more specific fMR imaging activation maps, we identified brain regions that were consistently activated across expression language tasks and comprehension tasks to create what we term an "expression fMR imaging map" and a "comprehension fMR imaging map," respectively. Expression language tasks included visual object naming, auditory responsive naming, and word list generation. Comprehension language tasks included visual responsive naming and sentence comprehension. To identify consistently activated regions, we used a conjoining procedure that identified all pixels in the brain exceeding the statistic threshold for two separate images (both expression or both comprehension). This procedure greatly reduced the probability of false positive results and is capable of detecting relatively low-amplitude, but consistent areas of increased brain activation. According to the Bayes theorem, the joint probability of observing significant pixels by chance on both images is equal to the product of the prior probability of chance activation on each; in this case, the chance probability is 0.063 and the joint probability is less than 0.004 (Pearson $r=0.2$ ). Two functional images were overlaid for each expression or comprehension map produced.

\section{Language Tasks}

All language tasks that required responses were performed covertly while the patient lay inside the MR magnet to minimize head movements.

Visual Object Naming. The patients were asked to name objects presented in the form of line drawings from the Boston Naming Test $^{19}$ (a standardized test of object naming with well established norms). Visual object naming is a robust activator of both essential and nonessential language cortices ${ }^{26}$ especially of the Broca area. ${ }^{5}$ Visual object naming is one of the most widely used tests for intraoperative mapping of eloquent cortices, because failure to complete the task (anomia) is one of the most common symptoms of aphasia. ${ }^{26}$

Word Generation. Patients were asked to generate lists of words beginning with a certain letter or belonging to a certain category (for example, animals). Word generation has been shown to be a robust activator of the frontal language areas. ${ }^{9}$

Auditory Responsive Naming. For this task patients were required to name objects that had been described to them. For example, the patient may have heard, "tall pink bird" and would be expected to think of a flamingo. Auditory responsive naming has also been shown to activate language areas in the frontal lobe. ${ }^{4}$

These first three tasks were considered expression, or productive, tasks because they largely call upon productive linguistic systems and activate frontal lobe language areas.

Visual Responsive Naming. This task is similar to auditory responsive naming, except that the description of the object is presented visually. Instead of hearing the phrase "tall pink bird," patients must read this description and think of the response.

Sentence Comprehension. Patients were presented with a pair of sentences that were identical in all respects, except for one word, which was replaced with either a synonym or a different word. The patients were instructed to listen to each pair of sentences and decide whether the two sen- tences had the same literal meaning. A previous study has demonstrated that this task is a strong activator of brain regions involved in semantic processing, near the perisylvian cortices. ${ }^{10}$

These final two tasks were considered comprehension tasks since they tax the semantic linguistic systems and activate posterior language sites more than the other three tasks described.

\section{Electrocortical Stimulation Mapping}

Intraoperatively, following craniotomy and dural reflection, the patient was awakened under the care of the operating room anesthesiologist and ESM was performed to localize language function. Due to time limitations, in most patients, only visual object naming was used for ESM because it is a robust activator of both essential and nonessential language cortices and because failure to perform this task (anomia) is one of the most common symptoms of aphasia. Once the patient was able to name pictures accurately and consistently, ESM of language function was performed using a bipolar electrode (5-mm interelectrode distance) and stimulator (S-12; Grass Instruments, Quincy, MA). While the patient named objects, cortical areas were stimulated and the patient was observed for language disruption. Stimulations consisted of 5-second trains beginning at $4 \mathrm{~mA}$, increasing in $2-\mathrm{mA}$ increments to a maximum of either $16 \mathrm{~mA}$, or $2 \mathrm{~mA}$ below the threshold for inducing afterdischarges-whichever is lower. Afterdischarge activity was monitored continuously during ESM. If afterdischarges were detected following stimulation, trials were repeated at a lower current. An area was considered nonessential to language if stimulation up to $16 \mathrm{~mA}$ did not affect language task performance (referred to herein as "clear ESM sites"). All other errors were noted and corresponding cortical areas were tagged as involved in language. In this report, those ESM sites that interrupted language function when stimulated are referred to as "ESM language sites."

\section{Comparison of Maps Generated by fMR Imaging and ESM}

Cortical extractions of each patient's brain were used as a common space for comparing different mapping modalities. Fast spin-echo images of each patient's brain were used to extract cortical surfaces for each patient. Each image was corrected for radiofrequency nonuniformity. ${ }^{33} \mathrm{~A}$ three-dimensional active surface algorithm was used to generate an external cortical surface mesh for each patient. ${ }^{20}$ Functional MR imaging activations were aligned with the cortical extractions by aligning the coplanar high-resolution EPI images by rigid body transformations with the fast spin-echo images used to create the cortical extraction. This transformation was done based on the principal of maximizing mutual information. ${ }^{21}$ Electrocortical stimulation maps were projected onto the cortical extraction by matching sulcal landmarks on the cortical extraction with the photograph of the exposed cortex covered by ESM tags and, subsequently, warping the image onto the surface (Fig. 1). ${ }^{28}$

\section{Matching Criteria}

Three different matching criteria were used for determin- 


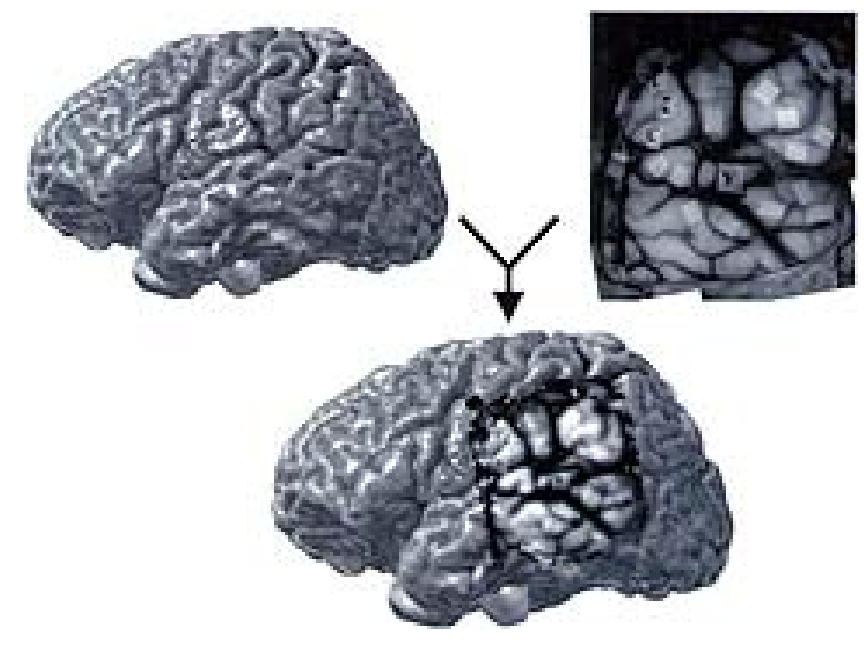

FIG. 1. Projection of ESM onto the cortical surface. To compare ESM, which provides a surface map, with fMR imaging activations, which are tomographic, the two maps were warped into a common space: a cortical model of each patient. Functional MR imaging activations were aligned with the cortical model by using rigid body transformation to align coplanar high-resolution EPI images with the MR image used to create the surface model. Electrocortical stimulation maps were warped onto the surface of the cortical model by matching sulcal landmarks on the cortical surface and the intraoperative photograph of the ESM. This warping technique accounts for brain shift that may occur intraoperatively.

ing whether ESM sites colocalized with or matched fMR imaging activations (Fig. 2). The first and most stringent criterion was that ESM sites must directly overlap with fMR imaging activations to be considered colocalized (Fig. 2 upper left). The second criterion required that fMR imaging activations either directly overlap with ESM sites or be located in the adjacent sulcus (Fig. 2 upper right). This criterion was based on the fact that BOLD fMR imaging activations are often centered within sulci and, therefore, may not be expected to colocalize directly with other maps. ${ }^{6,28}$ The third, least stringent, criterion considered fMR imaging activations and ESM sites to be colocalized if the two overlapped, if the fMR imaging activations were in the adjacent sulcus, or if these activations were deep within the gyrus that contained the ESM site (Fig. 2 lower left).

The effect of assuming different radii of activation of ESM was also assessed. Haglund and colleagues ${ }^{12,13}$ reported that resections located within $1 \mathrm{~cm}$ of ESM language sites are associated with deficits, and that the area affected by bipolar cortical stimulation increases with stimulus intensity and may extend several millimeters. These results suggest that ESM language localization, while relatively precise, ${ }^{25}$ may identify language sites that are not located precisely at the point of stimulation, but a small distance $(\leq$ $1 \mathrm{~cm}$ ) away. Consequently, we believed that it was important to assess how different ESM radii may affect the sensitivity and specificity of fMR imaging. The following three different radii were used: $2.5 \mathrm{~mm}$ (based on the interelectrode distance of the bipolar electrode), $5 \mathrm{~mm}$, and $10 \mathrm{~mm}$ (based on the reported findings of Haglund, et al. ${ }^{12}$ ) (Fig. 2 lower right).

Sensitivity was defined as the percentage of ESM language sites that were associated with fMR imaging activa-

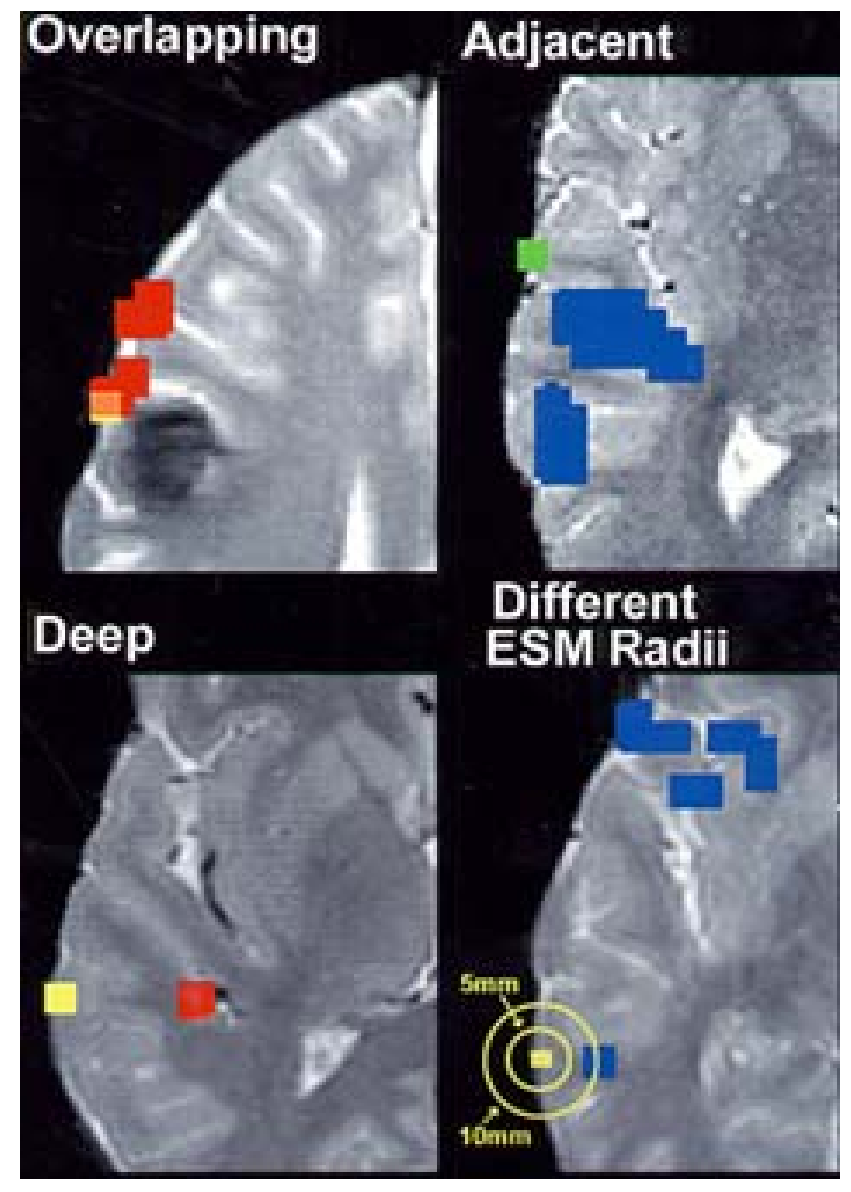

FIG. 2. Different matching criteria and ESM radii that were investigated. To determine the effect of different matching criteria on the sensitivity and specificity of fMR imaging in predicting ESM language sites, the sensitivity and specificity were calculated using different criteria for matching ESM and fMR imaging. The yellow and green boxes on the cortical surface represent essential and clean ESM sites, respectively. The blue and red boxes depict comprehension and expression fMR imaging activations, respectively. Upper Left: Overlap is the strictest criterion, requiring that ESM sites (at any given radius) directly overlap with observed fMR imaging activations. Upper Right: Using the adjacent criterion, fMR imaging and ESM are considered to be matching, or correlating, if fMR imaging activations are observed in the sulcus adjacent to the ESM language site. Lower Left: The most lenient criterion is to consider ESM and fMR imaging to be matching, or correlated, if fMR imaging activations were found deep with respect to the ESM language site. Lower Right: The effect of changing the radius of influence of ESM was also investigated. Three different radii were tested: $2.5 \mathrm{~mm}$ (represented by the central box), $5 \mathrm{~mm}$, and $10 \mathrm{~mm}$. In the example shown, the activations would be considered a direct overlap if the ESM site is viewed at a radius of $10 \mathrm{~mm}$, but not if it is viewed at a radius of 2.5 or $5 \mathrm{~mm}$. At a radius of 2.5 or $5 \mathrm{~mm}$, the fMR imaging activations shown here are considered to lie deep with respect to the ESM site.

tions. Specificity was defined as the percentage of clear ESM sites that did not have associated fMR imaging activations. The true-positive rate was defined as the proportion of cortical areas that were associated with fMR imaging activations that also tested positive for language by ESM. The true-negative rate was defined as the fraction of cortical ar- 


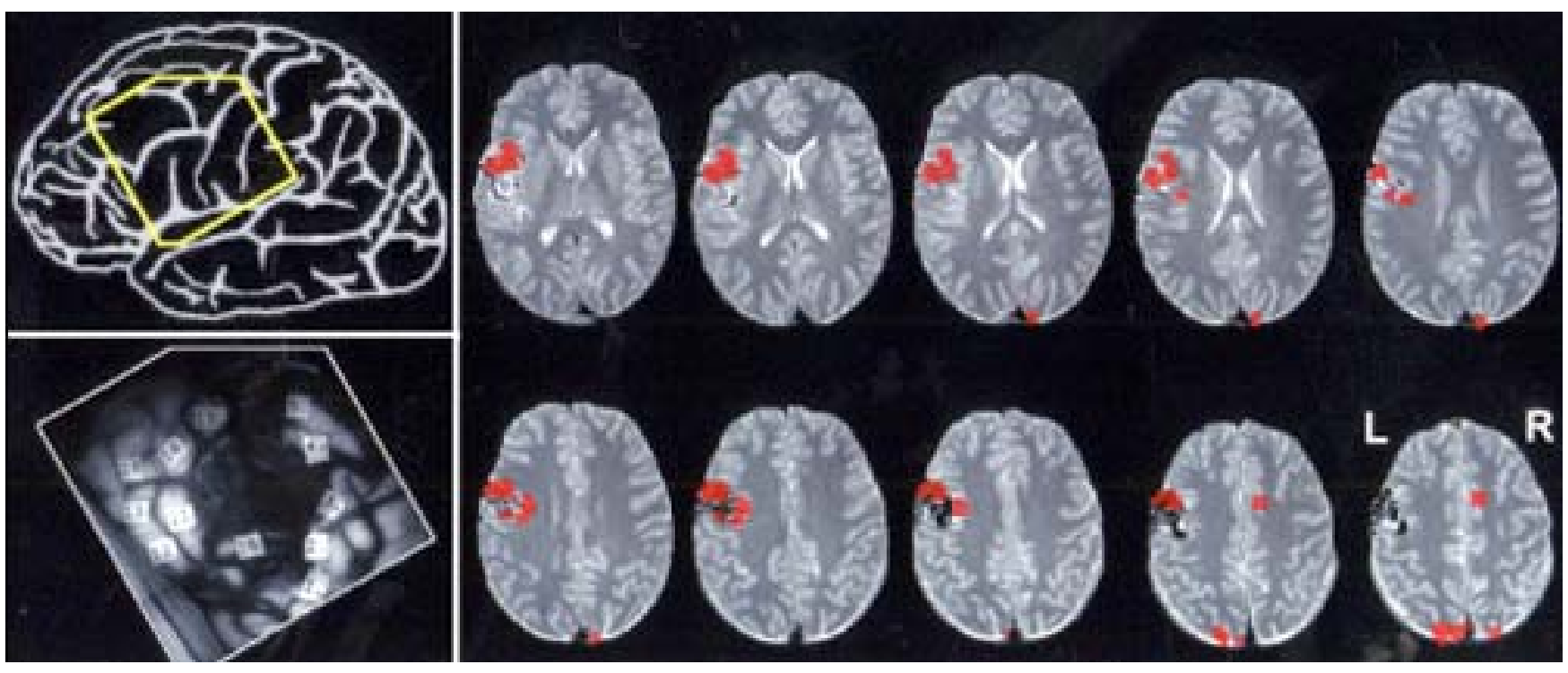

FIG. 3. Functional MR imaging activations adjacent to AVMs. Significant fMR imaging activations were commonly identified adjacent to AVMs. Activations were never identified within the vascular lesions. Upper Left: Schematic drawing of a brain showing the area mapped by ESM outlined in yellow. Lower Left: Intraoperative photograph demonstrating ESM of language function. Double-digit numbers indicate essential language areas. Single-digit numbers indicate the motor strip map. Significant ESM language sites were identified anterior and inferior to the AVM intraoperatively. Right: Functional MR imaging activations are qualitatively similar to electrocortical stimulation maps. Expression fMR imaging activations are shown overlayed on structural images, concentrated in areas anterior and inferior to the AVM, which is qualitatively similar to the results shown by ESM.

eas that were not associated with fMR imaging activations that also tested negative for language by ESM.

\section{Results}

Robust fMR imaging activations were identified in all patients, regardless of the type or size of their lesion. In Fig. 3 significant and extensive fMR imaging activations are shown directly adjacent and anterior to an AVM that measured approximately $3 \mathrm{~cm}$ at its largest diameter. Although activations were never identified within a vascular malformation, activity-related signals were consistently identified near pathological tissue. Activations always occurred in anatomically predictable regions - the left inferior frontal gyrus and the left posterior superior temporal sulcus - and corresponded with the results of ESM, as later described (Fig. 3).

At least six language sites in each patient were evaluated intraoperatively by using ESM. In general, we found that fMR imaging can be used reliably as a predictive tool for intraoperative ESM language sites. Expression fMR imaging maps consistently identified frontal lobe language sites, and comprehension fMR imaging maps accurately predicted ESM in both frontal and temporoparietal lobe regions (Fig. 4 and Table 1). In general, increasing the ESM radius and loosening the criteria for fMR imaging-ESM matches increased the sensitivity of fMR imaging, although it also decreased its specificity (Table 1).

\section{Expression fMR Imaging Maps}

We identified a strong and statistically significant relationship between expression fMR imaging activations and
ESM $\left(\chi^{2}=14.592-25.469, p<0.0005\right.$ [99 sites] for all comparisons at ESM radii of $2.5,5$, and $10 \mathrm{~mm}$ and the three different matching criteria described in Clinical Material and Methods). The association between expression fMR imaging maps and ESM language sites was strongest in the frontal lobe, near the inferior frontal gyrus (the Broca area). In the frontal lobe, the association of expression fMR imaging activations with positive ESM sites was significant across all ESM radii and matching criteria $\left(\chi^{2}=7.646-17.5\right.$, $p<0.0005-p<0.006$ [30 sites], significance depended on ESM radius and matching criteria). In the temporoparietal lobe region (the Wernicke area), no significant associations were identified $\left(\chi^{2}=1.285-3.122, \mathrm{p}>0.08\right.$ [69 sites] for all comparisons).

In the frontal lobe, sensitivity and specificity ranged from 100 and $44.4 \%$, respectively, to 76.2 and $77.8 \%$, respectively, depending on the ESM radius and the matching criterion used (Fig. 4 and Table 1). In general, increasing the ESM radius and loosening the criteria for a match between fMR imaging and ESM tended to both increase the sensitivity and decrease the specificity of fMR imaging. At the smallest ESM radius of $2.5 \mathrm{~mm}$ and the strictest matching criterion of overlapping ESM with fMR imaging activation, the sensitivity and specificity of expression fMR imaging tasks in the frontal lobe were 76.2 and $77.8 \%$, respectively. By increasing the ESM radius to $5 \mathrm{~mm}$, but still retaining the strict overlap criterion, a significant increase in sensitivity was achieved (100\%) with a modest cost to the specificity $(66.7 \%)$ (Fig. 5). Using these criteria, the true-positive rate in the frontal lobe was $87.5 \%$, with a corresponding falsepositive rate of $12.5 \%$ (see Table 1 for true-positive rates for other criteria). Given the same criteria, the true-negative rate in the frontal lobe was $100 \%$, with a false-negative rate 
fMRI of Expression Tasks

\section{All Exposed Cortices}
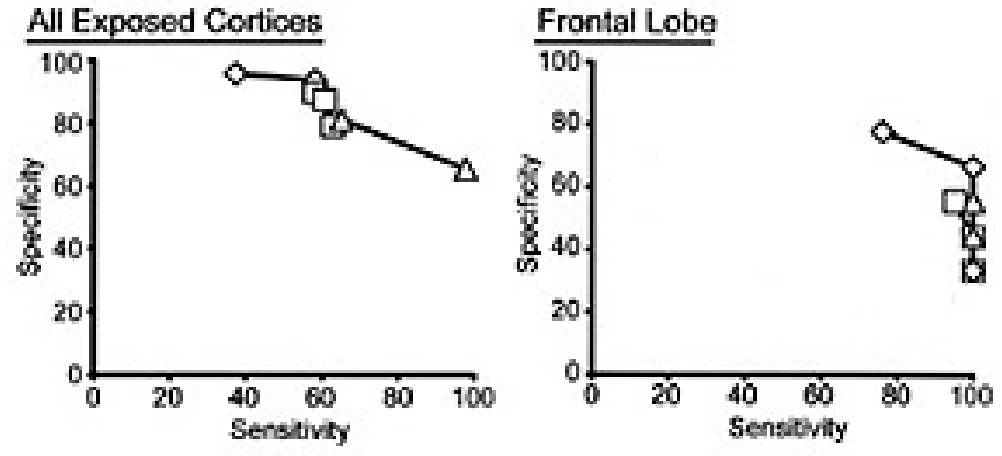

fMRI of Comprehension Tasks All Exposed Cortices
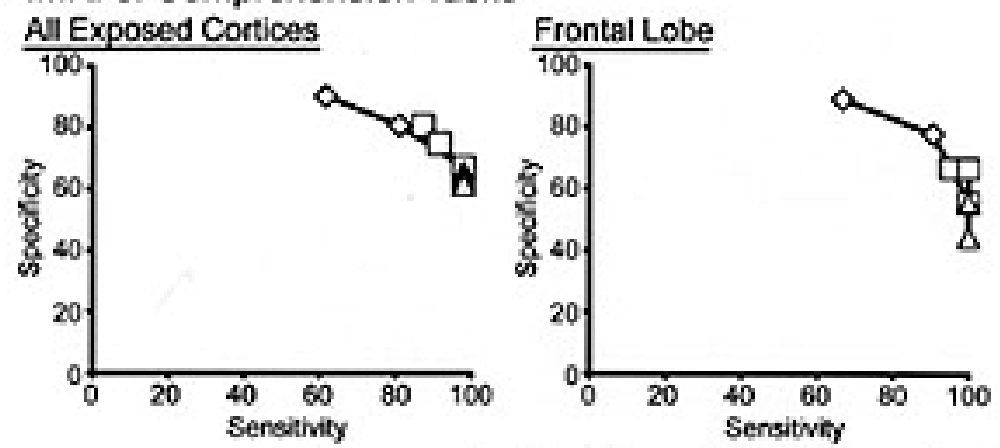

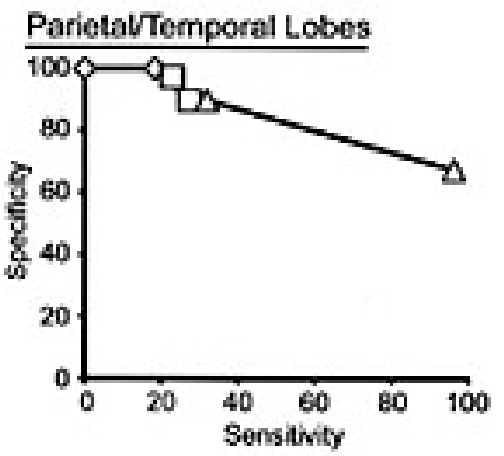

FIG. 4. Graphs plotting specificity versus sensitivity of fMR imaging (fMRI) for different matching criteria in all exposed cortices in the frontal lobe and the temporoparietal lobe region. An ideal clinical test would produce $100 \%$ sensitivity and $100 \%$ specificity and, therefore, be concentrated in the upper right region of each graph. In general, all data points tend to be concentrated in the upper right region of each graph (except for expression fMR imaging tasks in the temporoparietal lobe region), indicating that preoperative fMR imaging is, in fact, a good predictor of intraoperative ESM. In each graph, three points are shown for each matching criteria used, representing the three different ESM radii used. Diamonds represent overlap; squares, adjacent; and triangles, deep.

of $0 \%$. As stated earlier, increases in sensitivity were also achieved by loosening the matching criterion, but at a greater cost to specificity (see Table 1 for statistics covering all permutations of comparisons). Because the association of expression fMR imaging tasks with temporal and parietal lobe ESM language sites was not significant, we do not present the sensitivity and specificity values here, but include them in Table 1 for completeness.

\section{Comprehension fMR Imaging Maps}

We also identified a strong and statistically significant relationship between comprehension fMR imaging activations and ESM sites $\left(\chi^{2}=14.625-43.405, \mathrm{p}<0.0005\right.$ [99 sites] for all comparisons, regardless of ESM radius or the matching criterion used). Unlike expression fMR imaging maps, significant relationships were identified both in the frontal lobe $\left(\chi^{2}=7.778-17.5, \mathrm{p}<0.005-\mathrm{p}<0.0005[30\right.$ sites] for all comparisons, depending on ESM radius and the matching criterion used) and in the temporal and parietal lobes $\left(\chi^{2}=15.918-26.541, \mathrm{p}<0.0005\right.$ [69 sites] for all comparisons, regardless of the ESM radius or the matching criterion used).

Once again, increasing ESM radius and loosening matching criterion both increased sensitivity and decreased specificity (Fig. 4 and Table 1). Using the strictest criteria (ESM radius of $2.5 \mathrm{~mm}$ and overlapping fMR imaging acti- vation and ESM language site), the sensitivity and specificity in the frontal lobe were 66.7 and $88.9 \%$, respectively, and those in the temporal and parietal lobes were 57.7 and $90.7 \%$, respectively. Increasing the ESM radius to 10 $\mathrm{mm}$ increased the sensitivity to 100 and $96.2 \%$ in the frontal and temporoparietal lobe regions, respectively, and decreased the specificity to 55.6 and $69.8 \%$, respectively (Table 1 and Fig. 5). The corresponding true-positive rates were 84 and $65.8 \%$, respectively, and the false-positive rates were 16 and $34.2 \%$, respectively. The true-negative rates were 100 and $96.8 \%$, respectively, and the false-negative rates were 0 and $3.2 \%$, respectively. In the temporoparietal lobe region, there was no clear advantage with respect to sensitivity and specificity of using different matching criteria; increasing the ESM radius resulted in a better combination of sensitivity and specificity than loosening the matching criterion (Table 1).

\section{Specific Disease Populations}

To address the question of whether fMR imaging can be used as a predictive tool in different subpopulations of patients (patients harboring AVMs and those harboring cavernous angiomas), we also investigated the fMR imaging and ESM relationships in each subgroup of patients. Because the criterion of directly overlapping fMR imaging activations and ESM language sites was sufficient to identify 
significant associations in the entire population studied, we only investigated associations by using this strictest matching criterion.

Patients With AVMs. In the population of patients harboring AVMs expression fMR imaging activations were significantly related to $\mathrm{ESM}\left(\chi^{2}=7.607,20.488\right.$, and 12.438 , and $\mathrm{p}<0.006, \mathrm{p}<0.0005$, and $\mathrm{p}<0.0005$ for $\mathrm{ESM}$ radii of $2.5,5$, and $10 \mathrm{~mm}$, respectively [59 sites]). When analyzed according to lobes, significant associations between expression fMR imaging activations and ESM were identified at an ESM radius of $5 \mathrm{~mm}$ in both frontal and temporoparietal lobe regions $\left(\chi^{2}=10.588\right.$ and 7.131, and $\mathrm{p}<$ 0.001 and $p<0.008$, respectively [20 sites]). Sensitivity and specificity of expression fMR imaging activations in the frontal lobe were 100 and $60 \%$, respectively, and those in the temporoparietal lobe region were 26.7 and $100 \%$, respectively, at an ESM radius of $5 \mathrm{~mm}$. We can infer from these sensitivity and specificity values that expression fMR imaging maps may still be useful in patients with AVMs. As in the general population studied, however, the usefulness is limited to the frontal lobe. Furthermore, the criterion that produced the best combination of sensitivity and specificity in the frontal lobe in the general patient population produces the best results in patients harboring AVMs.

Comprehension fMR imaging activations in patients harboring AVMs were also strongly associated with whether a cortical area tested positive for language by ESM $\left(\chi^{2}=\right.$ $22.339,28.044$, and 38.529 at ESM radii of 2.5, 5, and 10 $\mathrm{mm}$, respectively, $\mathrm{p}<0.0005$ for all radii). In the temporoparietal lobe regions, significant associations were observed at all ESM radii $\left(\chi^{2}=18.72,16.787\right.$, and 25.76 for ESM radii of $2.5,5$, and $10 \mathrm{~mm}$, respectively, $\mathrm{p}<0.0005$ for all comparisons). The sensitivity and specificity for an ESM radius of $2.5 \mathrm{~mm}$ were 52.6 and $100 \%$, respectively, those for an ESM radius of $5 \mathrm{~mm}$ were 89.3 and $68.4 \%$, and those for an ESM radius of $10 \mathrm{~mm}$ were 100 and $75 \%$, respectively. In the frontal lobe, the association was significant at $\mathrm{ESM}$ radii of 5 and $10 \mathrm{~mm}\left(\chi^{2}=7.937\right.$ and 10.588 , respectively, $\mathrm{p}<0.005$ for both radii), but not at a radius of 2.5 $\mathrm{mm}$. The sensitivity and specificity at ESM radii of 5 and $10 \mathrm{~mm}$ were 86.7 and $80 \%$, and 100 and $60 \%$, respectively. These sensitivity and specificity values demonstrate that comprehension fMR imaging activations can be used just as reliably in populations of patients with AVMs as in the general patient population investigated in this study. Moreover, the criteria that produced the best combination of sensitivity and specificity in the general population studied (that is, an ESM radius of $10 \mathrm{~mm}$ and the overlapping matching criterion) also produced the best values in the population of patients with AVMs.

Patients With Cavernous Angiomas. In the population of patients with cavernous angiomas, a significant relationship of both expression and comprehension fMR imaging activations and ESM language sites was also observed. For expression fMR imaging activations, significant associations were only observed at ESM radii of 2.5 and $5 \mathrm{~mm}\left(\chi^{2}=\right.$ 8.661 and 7.552, and $\mathrm{p}<0.003$ and $\mathrm{p}<0.006$, respectively [32 sites]). When analyzed according to the lobe under investigation, expression fMR imaging activations were significantly associated with ESM language sites only in the frontal lobe and only at ESM radii of 2.5 and $5 \mathrm{~mm}$ $\left(\chi^{2}=6.667\right.$ and 6.429 , respectively, $\mathrm{p}=0.01$ for both radii
TABLE 1

Sensitivity and specificity, as well as true-positive and truenegative rates of fMR imaging for predicting language cortices*

\begin{tabular}{|c|c|c|c|c|c|}
\hline \multirow[b]{2}{*}{ Matching Criterion } & \multirow[b]{2}{*}{$\begin{array}{l}\text { ESM } \\
\text { Radius } \\
(\mathrm{mm})\end{array}$} & \multirow{2}{*}{$\begin{array}{c}\text { Sensi- } \\
\text { tivity } \\
(\%)\end{array}$} & \multirow{2}{*}{$\begin{array}{c}\text { Speci- } \\
\text { ficity } \\
(\%)\end{array}$} & \multicolumn{2}{|c|}{ Finding $(\%)$} \\
\hline & & & & $\begin{array}{l}\text { True } \\
\text { Positive }\end{array}$ & $\begin{array}{l}\text { True } \\
\text { Negative }\end{array}$ \\
\hline \multicolumn{6}{|c|}{$\begin{array}{l}\text { expression fMR imaging activations } \\
\text { frontal lobe }(30 \text { sites })\end{array}$} \\
\hline \multirow[t]{3}{*}{ overlap } & 2.5 & 76.2 & 77.8 & 88.9 & 58.3 \\
\hline & 5 & 100 & 66.7 & 87.5 & 100 \\
\hline & 10 & 100 & 33.3 & 77.8 & 100 \\
\hline \multirow[t]{3}{*}{ adjacent } & 2.5 & 95.2 & 55.6 & 83.3 & 83.3 \\
\hline & 5 & 100 & 44.4 & 80.8 & 100 \\
\hline & 10 & 100 & 33.3 & 77.8 & 100 \\
\hline \multirow[t]{3}{*}{ deep } & 2.5 & 100 & 44.4 & 80.8 & 100 \\
\hline & 5 & 100 & 44.4 & 80.8 & 100 \\
\hline & 10 & 100 & 55.6 & 84 & 100 \\
\hline \multicolumn{6}{|c|}{ parietotemporal lobe region ( 69 sites) } \\
\hline \multirow[t]{3}{*}{ overlap } & 2.5 & 0 & 100 & NA & 63.9 \\
\hline & 5 & 18.2 & 100 & 100 & 68.4 \\
\hline & 10 & 27.3 & 89.7 & 60 & 68.6 \\
\hline \multirow[t]{3}{*}{ adjacent } & 2.5 & 22.7 & 97.4 & 83.3 & 69.1 \\
\hline & 5 & 22.7 & 97.4 & 83.3 & 69.1 \\
\hline & 10 & 27.3 & 89.7 & 60 & 68.6 \\
\hline \multirow[t]{3}{*}{ deep } & 2.5 & 31.8 & 89.7 & 63.6 & 70 \\
\hline & 5 & 31.8 & 89.7 & 63.6 & 70 \\
\hline & 10 & 96.2 & 67.4 & 64.1 & 96.7 \\
\hline \multicolumn{6}{|c|}{$\begin{array}{l}\text { comprehension fMR imaging activations } \\
\text { frontal lobe ( } 30 \text { sites) }\end{array}$} \\
\hline \multirow[t]{3}{*}{ overlap } & 2.5 & 66.7 & 88.9 & 93.3 & 53.3 \\
\hline & 5 & 90.5 & 77.8 & 90.5 & 77.8 \\
\hline & 10 & 100 & 55.6 & 84 & 100 \\
\hline \multirow[t]{3}{*}{ adjacent } & 2.5 & 95.2 & 66.7 & 87 & 85.7 \\
\hline & 5 & 100 & 66.7 & 87.5 & 100 \\
\hline & 10 & 100 & 55.6 & 84 & 100 \\
\hline \multirow[t]{3}{*}{ deep } & 2.5 & 100 & 55.6 & 84 & 100 \\
\hline & 5 & 100 & 55.6 & 84 & 100 \\
\hline & 10 & 100 & 44.4 & 80.8 & 100 \\
\hline \multicolumn{6}{|c|}{ parietotemporal lobe region (69 sites) } \\
\hline \multirow[t]{3}{*}{ overlap } & 2.5 & 57.7 & 90.7 & 78.9 & 78 \\
\hline & 5 & 73.1 & 81.4 & 70.4 & 83.3 \\
\hline & 10 & 96.2 & 69.8 & 65.8 & 96.8 \\
\hline \multirow[t]{3}{*}{ adjacent } & 2.5 & 80.8 & 83.7 & 75 & 87.8 \\
\hline & 5 & 84.6 & 76.7 & 68.8 & 89.2 \\
\hline & 10 & 96.2 & 69.8 & 65.8 & 96.8 \\
\hline \multirow[t]{3}{*}{ deep } & 2.5 & 96.2 & 67.4 & 64.1 & 96.6 \\
\hline & 5 & 96.2 & 65.1 & 62.5 & 96.6 \\
\hline & 10 & 96.2 & 65.1 & 62.5 & 96.6 \\
\hline
\end{tabular}

* Matching criterion refers to the criterion used to determine whether ESM and fMR imaging were considered to match. The ESM radius refers to the assumed radius of influence of direct cortical stimulation. See Fig. 2 and text for further details and definitions. Note that corresponding falsepositive rates equal the difference between the unity and the true-positive rate and false-negative rates equal the difference between unity and the truenegative rate. Abbreviation: $\mathrm{NA}=$ not applicable.

[10 sites]). The sensitivity and specificity in the frontal lobe at ESM radii of 2.5 and $5 \mathrm{~mm}$ were 83.3 and $100 \%$, and 100 and $75 \%$, respectively. These values for sensitivity and specificity are once again consistent with the results obtained in the general population studied. Comprehension fMR imaging activations were significantly associated with ESM language sites at all radii $\left(\chi^{2}=9.791,11.567\right.$, and 8.3, and $\mathrm{p}<0.002, \mathrm{p}<0.001$, and $\mathrm{p}<0.004$ at radii of 2.5, 5, and $10 \mathrm{~mm}$, respectively). This association was consistently found in both the frontal lobe and the temporoparietal lobe regions. In the frontal lobe, comprehension fMR imag- 


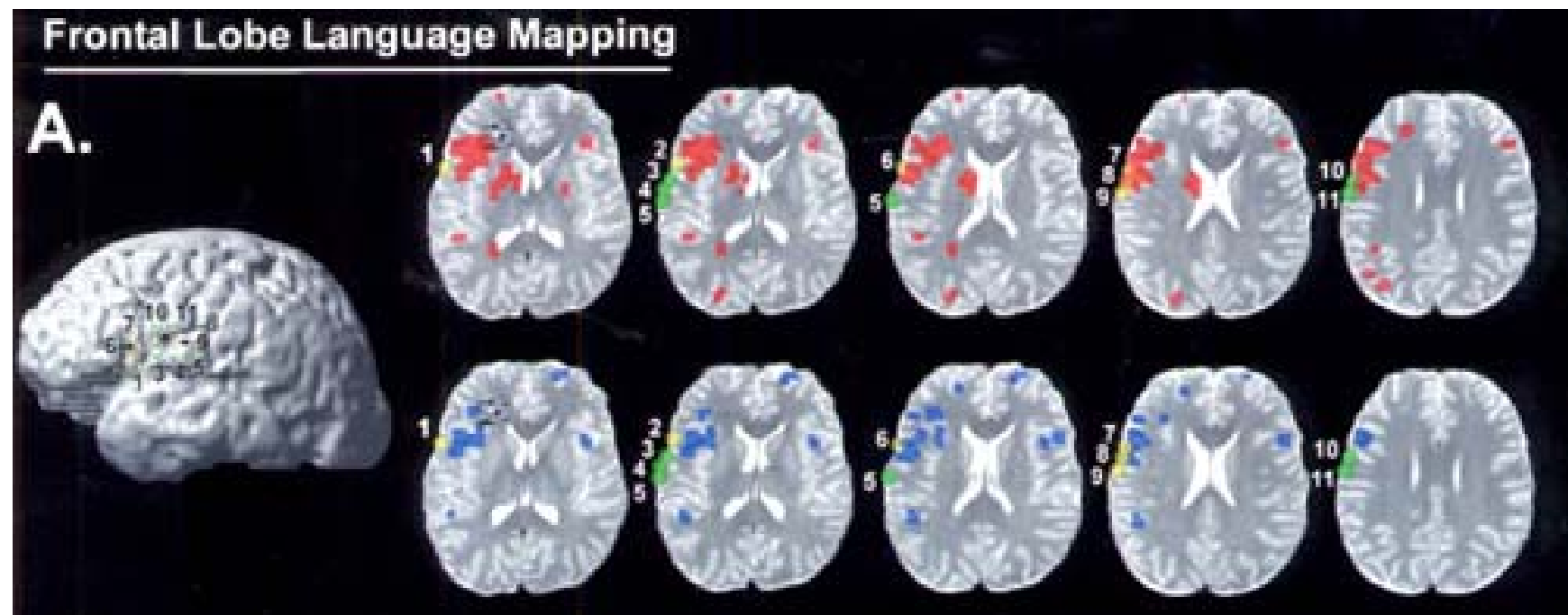

\section{Parietal/Temporal Lobe Language Mapping}

B.
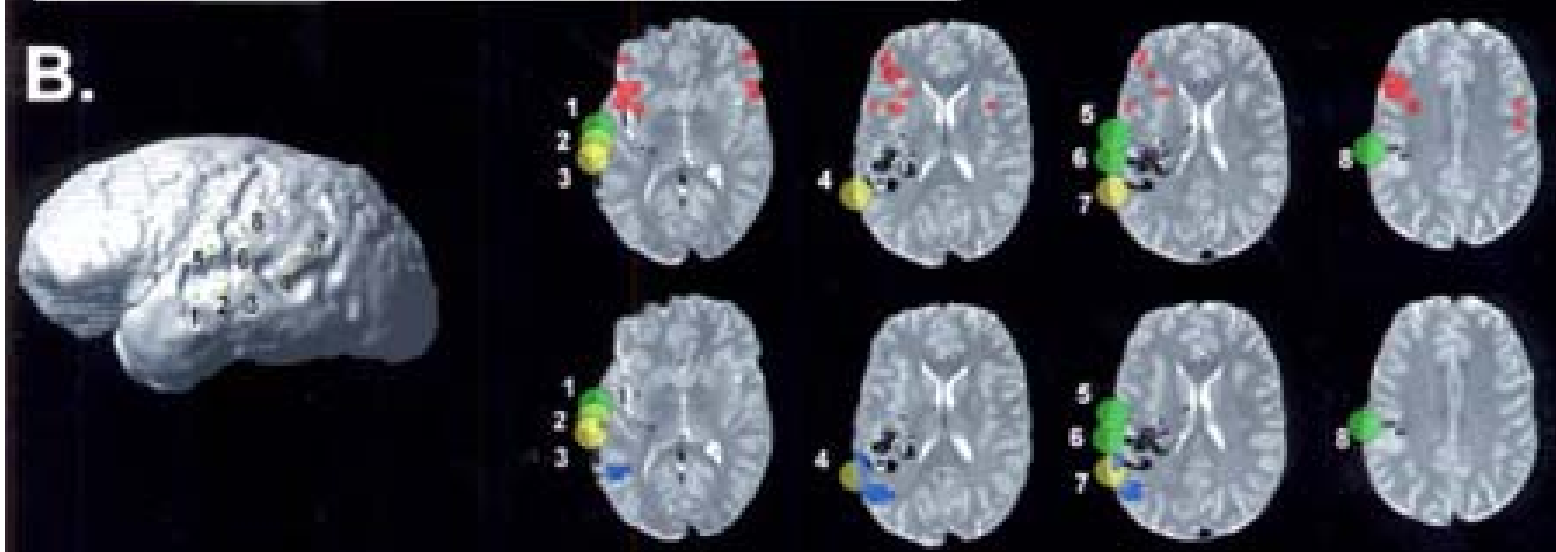
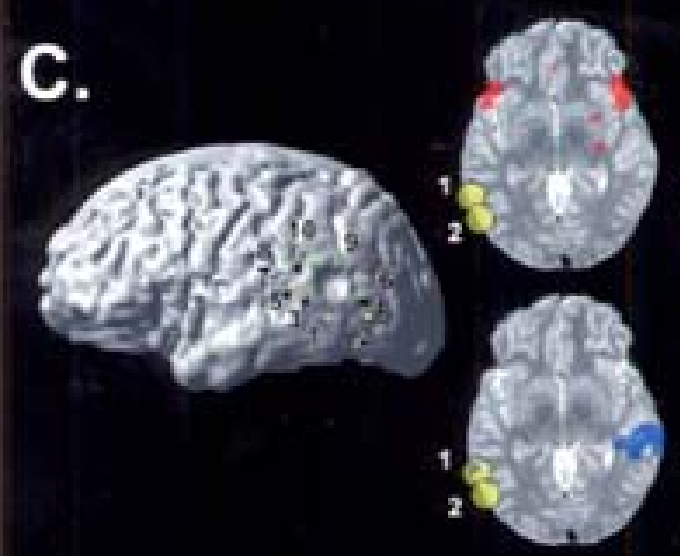
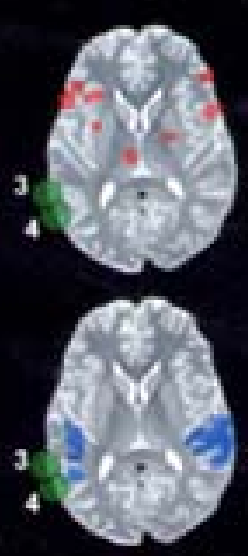
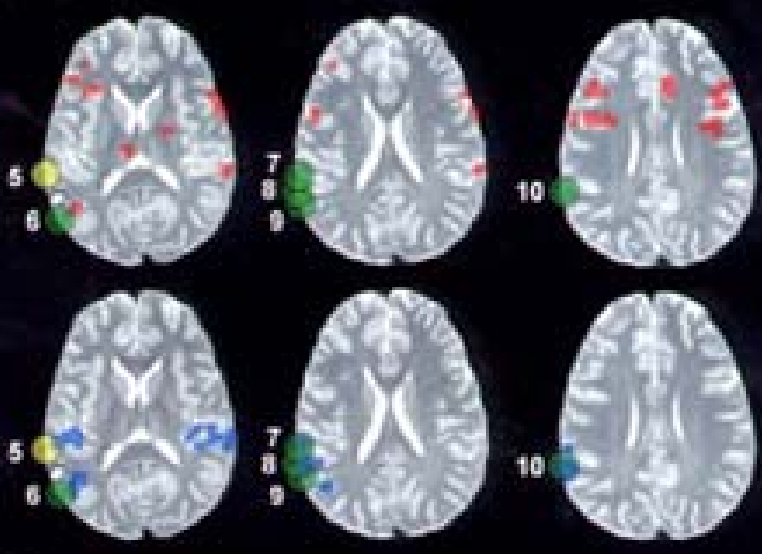

FIG. 5. Examples of language mapping in the frontal lobe (A) and the combined temporal and parietal lobes (B). Yellow and green circles represent numbered ESM language sites and clean ESM sites, respectively. Red boxes represent expression fMR imaging activations. Blue boxes represent comprehension fMR imaging activations. The frontal lobe slices are shown with an ESM radius of $5 \mathrm{~mm}$ (determined to produce the highest sensitivity with the least cost to specificity) and temporoparietal lobe slices are shown with an ESM radius of $10 \mathrm{~mm}$. A: For frontal lobe mapping, these brain slices demonstrate that red (expression) activations tend to overlap with,

FIG. 5. (continued) $\rightarrow$ 


\section{Preoperative fMR imaging of language in patients with AVMs}

ing activations were significantly associated with ESM language sites at ESM radii of 2.5 and $5 \mathrm{~mm}\left(\chi^{2}=6.667\right.$ and 6.429 , respectively, $\mathrm{p}<0.01$ for both [10 sites]). The sensitivity and specificity in the frontal lobe for comprehensive fMR imaging activations were 83.3 and $100 \%$, and 100 and $75 \%$ for ESM radii of 2.5 and $5 \mathrm{~mm}$, respectively. In the temporoparietal lobe regions, marginally significant associations were identified at all ESM radii $\left(\chi^{2}=3.956,5.238\right.$, and 4.023, and $\mathrm{p}<0.05, \mathrm{p}<0.03$, and $\mathrm{p}<0.05$ at ESM radii of $2.5,5$, and $10 \mathrm{~mm}$, respectively). The sensitivity and specificity for a radius of $2.5 \mathrm{~mm}$ were 71.4 and $73.3 \%$, those for a radius of $5 \mathrm{~mm}$ were 85.7 and $66.7 \%$, and those for a radius of $10 \mathrm{~mm}$ were 85.7 and $60 \%$, respectively.

\section{Individual Variability}

We also reviewed individual variability in the sensitivity and specificity of fMR imaging activations. In the results reported here for the general population of patients with vascular malformations, with respect to expression fMR imaging tasks, the best combination of sensitivity and specificity was observed with an ESM radius of $5 \mathrm{~mm}$ and the strict overlapping criterion. Furthermore we report that expression fMR imaging activations were only useful in predicting frontal lobe language sites. Using these criteria, we evaluated individual values for sensitivity and specificity in the three patients with frontal lobe lesions. The sensitivity and specificity for these individuals were 100 and $60 \%$ (11 sites), 100 and $100 \%$ (seven sites), and 100 and $75 \%$ (10 sites). For comprehension fMR imaging activations, we reported earlier that assuming an ESM radius of $10 \mathrm{~mm}$ and the strict overlapping criterion for activations produced the best combination of sensitivity and specificity. Because comprehension fMR imaging activations were found to be associated with ESM language sites regardless of the lobe examined, individual values for sensitivity and specificity were reviewed for all patients by using these criteria. The sensitivity and specificity values were as follows: 100 and $60 \%$ (11 sites), 100 and $66.7 \%$ (13 sites), 100 and 55.6\% (15 sites), 100 and $100 \%$ (six sites), 66.7 and $28.6 \%$ (10 sites; illustrated in Fig. 5C), 100 and 87.5\% (12 sites), 100 and $100 \%$ (seven sites), 100 and $50 \%$ (10 sites), 100 and 100\% (seven sites), and 100 and $75 \%$ (eight sites), respectively. Except for one case, these results clearly indicate that the criteria indicated (assumed to be an ESM radius of 10

or are adjacent to, essential (yellow) ESM sites, but avoid nonessential (green) ESM sites. Blue activations in the frontal lobe also appear predictive. B: In most cases of temporoparietal lobe mapping, such as the one illustrated here, comprehension fMR imaging activations matched well with ESM language sites (yellow) in the temporoparietal lobes and did not overlap with clean ESM sites (green). Very little expression fMR imaging activations are seen in the temporoparietal lobe region and the reason why such tasks as verbal object naming and word generation do not accurately predict language sites in these regions. C: These maps were obtained in the individual case in which preoperative fMR imaging was the least effective at accurately predicting whether a given cortical area would be involved in language function. In this case, only two of the three essential ESM sites overlapped with fMR imaging activations, and only two of seven of the clean ESM sites completely avoided fMR imaging activations. $\mathrm{mm}$ and the strict overlapping criterion) produce extremely powerful results for preoperative mapping and identification of all essential language sites (as determined by ESM). It is notable that the sensitivity and specificity values reported were relatively consistent across individuals, despite the fact that the number of ESM language sites varied considerably across individuals (total number of sites varied from six to 15) and the ratio of ESM language sites to clear ESM sites varied considerably across the population (from $7: 1$ to $4: 9)$.

\section{Discussion}

This is the first report in which it is demonstrated that preoperative fMR imaging can be used successfully to identify whether a given cortical area is essential for language (as determined by ESM) with extremely high sensitivity and acceptable specificity in patients with vascular malformations. We demonstrate that expression fMR imaging maps can best be used to identify potential essential language sites in the frontal lobe, whereas comprehension fMR imaging maps can be used to identify language sites in both potential frontal lobe and temporoparietal lobe regions.

\section{Perfusion-Related Signals in Patients With Vascular Malformations}

We demonstrate unequivocally that activity-related, perfusion-related signals can be observed in patients with vascular malformations, even when the activation is directly adjacent to the AVMs, despite the fact that vascular malformations, especially AVMs, are associated with abnormal blood flow (Figs. 3 and 5). Activations were seen regardless of the size or location of the AVM. Although signals were regularly observed adjacent to vascular malformations, no BOLD signals were observed within the vascular malformations due to a significant degree of susceptibility artifact within the pathological area. The susceptibility artifact is likely associated with the turbulent flow within these structures and resulting inhomogeneities in the magnetic field within the diseased sites.

The presence of activity-related, perfusion-related signals directly adjacent to AVMs were first reported by authors who used PET scanning with $\mathrm{H}_{2}{ }^{15} \mathrm{O}$ and were confirmed using intraoperative cortical mapping. ${ }^{17}$ Since then there have been several reports demonstrating fMR imaging activations adjacent to AVMs., ${ }^{1,2,32}$ The correlation of these perfusion-related signals with intraoperative cortical mapping, however, has remained unclear. Although perfusion-related signals have been correlated with intraoperative cortical maps previously in other patient populations, ${ }^{3,11}$ to date only a single case report has demonstrated the correlation between intraoperative cortical maps and perfusionrelated signals (as measured by PET scanning) in a patient harboring an AVM. ${ }^{17}$

\section{Sensitivity and Specificity of fMR Imaging}

The ideal mapping procedure should be both sensitive and specific: sensitive so that we can be confident that all essential language sites have been identified, and specific so that those areas that are not essential to language function do not test positively. It is important to consider sensitivity 
and specificity simultaneously. Although $100 \%$ sensitivity and $100 \%$ specificity would be ideal, given any clinical test a compromise must be made between the two. In mapping of essential language sites, it is paramount that all essential language sites are associated with a positive test (that is, high sensitivity) to minimize or eliminate false negative findings (that is, cortices not demonstrating fMR imaging activity but found to be essential by ESM). False-negative findings could possibly lead to resection of essential tissue, leaving patients aphasic. Consequently, maximizing sensitivity seems appropriate, even at the expense of reduced specificity or increased rate of false-positive findings. Although false-positive findings are also undesirable because they may discourage maximum resection, they can be checked for intraoperatively by directed, site-specific ESM.

Based on differences between ESM and fMR imaging methods, false-positive findings should be expected in any case. Whereas ESM is a disruption-based method, which identifies only those areas that are essential to language processing, fMR imaging is an activation-based method that identifies all regions of the brain that demonstrate activityrelated changes, whether those areas are essential or supplementary. Consequently, areas that appear negative for language when ESM is used may still demonstrate fMR imaging activations, producing false-positive results. Decreased specificity may also be expected because fMR imaging is a perfusion-based method and does not directly detect neuronal activity. Perfusion-related signals may not be restricted precisely to cortical areas with increased neuronal activity, thereby also producing false-positive results. In the worst example we observed (Fig. 5C), five of seven clean ESM sites were associated with fMR imaging activations and only two of seven ESM sites associated with fMR imaging activations were deemed essential language sites by ESM (corresponding to a false-positive rate of $71.4 \%$ in this case). In light of the limited specificity and the significant false-positive rates reported for preoperative fMR imaging, it is critical to verify preoperative fMR imaging language maps intraoperatively by using ESM.

The false-negative rate of preoperative fMR imaging for predicting whether a cortical area is essential for language (according to ESM), on the other hand, was $0 \%$ in most cases. The exception was in temporoparietal lobe regions and occurred when using comprehension preoperative fMR imaging maps, in which the false-negative rate was 3.2\%. False-negative findings represent cortical sites that were deemed essential for language processing by ESM, but did not have an overlapping BOLD fMR imaging signal. The extremely low false-negative rate is an expected result, considering that BOLD fMR imaging maps identify all language areas (both essential and supplementary), whereas ESM only identifies essential language sites. False-negative results may be due to several factors, including the imprecise neurovascular coupling in the spatial dimension, ${ }^{15}$ the fact that BOLD fMR imaging signals often emphasize signals in adjacent sulci rather than in gyri, ${ }^{6,28,29}$ and susceptibility artifacts in the temporal lobe, all of which may reduce the ability of BOLD signals to colocalize precisely with ESM language sites.

We report that both expression and comprehension fMR imaging activation maps can be used to predict the localization of language function by ESM with extremely high sensitivity and acceptable specificity. Specifically, we re- port that, assuming a radius of ESM influence of 5 and 10 $\mathrm{mm}$ for expression and comprehension fMR imaging activations, respectively, a sensitivity rate of 95 to $100 \%$ and a specificity rate of 65 to $70 \%$ can be achieved. The ESM radii reported here are consistent with previous reports of the sphere of influence of an electrocortical stimulation site. ${ }^{12,13}$ Haglund, et al., ${ }^{12}$ reported that resection farther than $1 \mathrm{~cm}$ from essential language sites in the temporal lobe is associated with full language recovery. Consistent with their findings, we report that in the temporal lobe, a radius of $10 \mathrm{~mm}$, or $1 \mathrm{~cm}$, must be assumed to achieve nearly $100 \%$ sensitivity. In the frontal lobe, however, we found that a smaller ESM radius $(5 \mathrm{~mm})$ can be used to achieve $100 \%$ sensitivity and still retain relatively high specificity. This is also consistent with reports that essential language sites are more concentrated in the frontal lobe than in the temporal lobe. ${ }^{26}$

The sensitivity and specificity of fMR imaging appears consistent across disease populations. Even after excluding the three patients who harbored cavernous angiomas from the study, we report a significant interaction of fMR imaging activations and ESM language sites in the remaining patients with AVMs. These findings indicate that preoperative fMR imaging mapping can still be used faithfully, even in the population of patients with AVMs, in which there are blood-flow abnormalities and limited MR imaging artifacts to predict whether cortical areas are essential for language processing. A full prospective study of patient outcomes, however, should still be conducted to validate the clinical utility of preoperative fMR imaging.

\section{Individual Variability}

In evaluating a clinical tool, it is not only important to determine the tool's usefulness for the general population studied but also for individuals to ensure that a few individuals do not carry the entire population toward significance. Despite a great degree of variability across patients with respect to the number and location of essential language sites and the size and location of the disease, the sensitivity and specificity of fMR imaging was remarkably consistent across patients. Of all patients studied, in only one individual was the fMR imaging sensitivity below $100 \%$ and specificity below $50 \%$, suggesting that these methods of mapping may not be adequate for every individual. This presents a potentially significant limitation for preoperative planning using fMR imaging. Nevertheless, this limitation can and should be accounted for by confirming potential language sites intraoperatively with the aid of ESM.

By combining preoperative fMR imaging with ESM, intraoperative mapping time may be reduced significantly by using site-specific cortical mapping. This assertion is based on the characteristics of preoperative fMR imaging maps relative to ESM, having a close to $0 \%$ false-negative rate and an approximately 30\% false-positive rate. Instead of having to use ESM to evaluate the entire cortical exposure for essential language sites, preoperative fMR imaging can direct the surgeon toward areas containing fMR imaging activations to determine whether the indicated cortices are essential or nonessential. Preoperative fMR imaging may also be considered for deciding whether to operate. We report the true-positive rate of fMR imaging (that is, the proportion of areas with fMR imaging activations that are actu- 


\section{Preoperative fMR imaging of language in patients with AVMs}

ally essential ESM language sites) to be 87.5 and $65.8 \%$ for expression and comprehension tasks, respectively. These numbers indicate that approximately two thirds to seven eighths of those sites associated with fMR imaging activity are essential language sites. A final significant advantage of integrating preoperative fMR imaging into mapping protocols is that the procedure is noninvasive and well tolerated.

\section{Task and Lobe Dependence of Sensitivity and Specificity}

Several studies have indicated that posterior language regions (including the superior and middle temporal gyri, the supramarginal gyrus, and the angular gyrus, located in the temporal and parietal lobes) are associated with lexicosemantic processing, whereas anterior language regions (posterior inferior frontal gyrus [the Broca area], located in the frontal lobe) are associated with morphosyntactic processing. We therefore expected preoperative fMR imaging activations to be task and lobe dependent, considering the language functions classically assigned to different language areas. Consistent with the aforementioned premise, we found that expression fMR imaging activations were very good at identifying language sites in the frontal lobe and that comprehension fMR imaging activations were very good at identifying language sites in the temporoparietal lobe regions. We should not expect tasks such as visual object naming, which primarily activated frontal lobe regions to be useful for identifying posterior language sites in the temporal and parietal lobes. We do report, however, that comprehension fMR imaging activations successfully identified frontal lobe language sites as defined by ESM. We believe this is due to the fact that one of the comprehension tasks (visual responsive naming) requires the patient to produce a response (the name of the object described). Thus, although it activates posterior language areas during the comprehension part of the task, it also activates the productive language center when the patient produces the response.

\section{Conclusions}

Preoperative fMR imaging offers high sensitivity and acceptable specificity in determining the location of essential language sites in both the frontal and temporoparietal lobe regions. The sensitivity and specificity is task and lobe dependent, however. To achieve the values of sensitivity and specificity that we have reported, a conjoining procedure should be used to identify relatively low-amplitude, but consistent, areas of increased brain activation. Although expression fMR imaging tasks can be used reliably to predict language sites in the frontal lobe, they cannot be used to predict sites in the temporal and parietal lobes, which classically have been associated with comprehension and semantic processing. To identify language sites in the temporal and parietal lobes reliably, comprehension-based fMR imaging activations must be used. Furthermore, to achieve high sensitivity and specificity, one must look for fMR imaging activations up to 5 to $10 \mathrm{~mm}$ away from the site in question.

The goal of the current study was to characterize the relationship between preoperative fMR imaging maps and electrocortical stimulation maps. In this study we did not investigate patient outcomes. Future studies should be directed toward characterizing the relationship between BOLD fMR imaging activations, extent of resection, and clinical outcomes to furnish a better definition of the role of preoperative fMR imaging in surgical planning, similar to what has been done for ESM. ${ }^{25}$ Although our results demonstrate a high correlation between fMR imaging and ESM, no firm conclusions can be drawn regarding the relationship between fMR imaging language mapping and clinical outcomes.

\section{Acknowledgments}

The authors thank Nina Molayem and Melissa Walker for their help with this study.

\section{References}

1. Alkadhi H, Kollias SS, Crelier GR, et al: Plasticity of the human motor cortex in patients with arteriovenous malformations: a functional MR imaging study. AJNR 21:1423-1433, 2000

2. Baumann SB, Noll DC, Kondziolka DS, et al: Comparison of functional magnetic resonance imaging with positron emission tomography and magnetoencephalography to identify motor cortex in a patient with an arteriovenous malformation. J Image Guid Surg 1:191-197, 1995

3. Bookheimer SY, Zeffiro TA, Blaxton T, et al: A direct comparison of PET activation and electrocortical stimulation mapping for language localization. Neurology 48:1056-1065, 1997

4. Bookheimer SY, Zeffiro TA, Blaxton TA, et al: Regional cerebral blood flow during auditory responsive naming: evidence for cross-modality neural activation. Neuroreport 9:2409-2413, 1998

5. Bookheimer SY, Zeffiro TA, Blaxton TA, et al: Regional cerebral blood flow during object naming and word reading. Hum Brain Mapp 3:93-106, 1995

6. Cannestra AF, Pouratian N, Bookheimer SY, et al: Temporal spatial differences observed by functional MRI and human intraoperative optical imaging. Cereb Cortex 11:773-782, 2001

7. Cohen MS: Parametric analysis of fMRI data using linear systems methods. Neuroimage 6:93-103, 1997

8. Cohen MS, Bookheimer SY: Localization of brain function using magnetic resonance imaging. Trends Neurosci 17:268-277, 1994

9. Cuenod CA, Bookheimer SY, Hertz-Pannier L, et al: Functional MRI during word generation, using conventional equipment: a potential tool for language localization in the clinical environment. Neurology 45:1821-1827, 1995

10. Dapretto M, Bookheimer SY: Form and content: dissociating syntax and semantics in sentence comprehension. Neuron 24: 427-432, 1999

11. FitzGerald DB, Cosgrove GR, Ronner S, et al: Location of language in the cortex: a comparison between functional MR imaging and electrocortical stimulation. AJNR 18:1529-1539, 1997

12. Haglund MM, Berger MS, Shamseldin M, et al: Cortical localization of temporal lobe language sites in patients with gliomas. Neurosurgery 34:567-576, 1994

13. Haglund MM, Ojemann GA, Blasdel GG: Optical imaging of bipolar cortical stimulation. J Neurosurg 78:785-793, 1993

14. Haglund MM, Ojemann GA, Hochman DW: Optical imaging of epileptiform and functional activity in human cerebral cortex. Nature 358:668-671, 1992

15. Lai S, Hopkins AL, Haacke EM, et al: Identification of vascular structures as a major source of signal contrast in high resolution 2D and 3D functional activation imaging of the motor cortex at 1.5T: preliminary results. Magn Reson Med 30:387-392, 1993

16. Latchaw RE, Hu X, Ugurbil K, et al: Functional magnetic resonance imaging as a management tool for cerebral arteriovenous malformations. Neurosurgery 37:619-626, 1995 
17. Leblanc R, Meyer E: Functional PET scanning in the assessment of cerebral arteriovenous malformations. Case report. J Neurosurg 73:615-619, 1990

18. Lurito JT, Lowe MJ, Sartorius C, et al: Comparison of fMRI and intraoperative direct cortical stimulation in localization of receptive language areas. J Comput Assist Tomogr 24:99-105, 2000

19. Kaplan E, Goodglass H, Weintraub S (eds): Boston Naming Test. Philadelphia: Lee \& Febiger, 1983

20. MacDonald D, Avis D, Evans AC: Multiple surface identification and matching in magnetic resonance images. Proc SPIE 2359: 160-169, 1994

21. Maes F, Collignon A, Vandermeulen D, et al: Multimodality image registration by maximization of mutual information. IEEE Trans Med Imaging 16:187-198, 1997

22. Maldjian J, Atlas SW, Howard RS II, et al: Functional magnetic resonance imaging of regional brain activity in patients with intracerebral arteriovenous malformations before surgical or endovascular therapy. J Neurosurg 84:477-483, 1996

23. McDonald JD, Chong BW, Lewine JD, et al: Integration of preoperative and intraoperative functional brain mapping in a frameless stereotactic environment for lesions near eloquent cortex. Technical note. J Neurosurg 90:591-598, 1999

24. Ogawa S, Menon RS, Tank DW, et al: Functional brain mapping by blood oxygenation level-dependent contrast magnetic resonance imaging. A comparison of signal characteristics with a biophysical model. Biophys J 64:803-812, 1993

25. Ojemann GA: Brain organizaton for language from the perspective of electrical stimulation mapping. Behav Brain Sci 6: 189-230, 1983

26. Ojemann GA: Functional mapping of cortical language areas in adults. Intraoperative approaches. Adv Neurol 63:155-163, 1993

27. Ojemann GA, Whitaker HA: The bilingual brain. Arch Neurol 35:409-412, 1978

28. Pouratian N, Bookheimer SY, O'Farrell AM, et al: Optical imaging of bilingual cortical representations. Case report. J Neurosurg 93:676-681, 2000

29. Pouratian N, Sicotte N, Rex D, et al: Spatial/temporal correlation of BOLD and optical intrinsic signals in humans. Magn Reson Med 47:766-776, 2002

30. Pujol J, Deus J, Losilla JM, et al: Cerebral lateralization of lan- guage in normal left-handed people studied by functional MRI. Neurology 52:1038-1043, 1999

31. Rutten GJ, van Rijen PC, van Veelen CW, et al: Language area localization with three-dimensional functional magnetic resonance imaging matches intrasulcal electrostimulation in Broca's area. Ann Neurol 46:405-408, 1999

32. Schlosser MJ, McCarthy G, Fulbright RK, et al: Cerebral vascular malformations adjacent to sensorimotor and visual cortex. Functional magnetic resonance imaging studies before and after therapeutic intervention. Stroke 28:1130-1137, 1997

33. Sled JG, Zijdenbos AP, Evans AC: A nonparametric method for automatic correction of intensity nonuniformity in MRI data. IEEE Trans Med Imaging 17:87-97, 1998

34. Spetzler RF, Martin NA: A proposed grading system for arteriovenous malformations. J Neurosurg 65:476-483, 1986

35. Woods RP, Cherry SR, Mazziotta JC: Rapid automated algorithm for aligning and reslicing PET images. J Comput Assist Tomogr 16:620-633, 1992

Manuscript received April 20, 2001.

Accepted in final form April 9, 2002.

Dr. Pouratian and Mr. Rex are supported, in part, by the National Institutes of General Medical Sciences Medical Scientist Training Program (Grant No. GM08042). Dr. Pouratian is also supported by a National Institutes of Mental Health National Research Service Award (Grant No. MH12773). Additional support was provided by research grants to Dr. Bookheimer (Grant No. RR12169 from the National Center for Research Resources) and Dr. Toga (Grant No. MH/NS52083 from the National Institutes of Mental Health). Support was also provided by the Brain Mapping Medical Research Organization, Brain Mapping Support Foundation, Pierson-Lovelace Foundation, The Ahmanson Foundation, Tamkin Foundation, Jennifer Jones-Simon Foundation, Capital Group Companies Charitable Foundation, Robson Family, and the Northstar Fund.

Address reprint requests to: Arthur W. Toga, Ph.D., Laboratory of Neuro Imaging, 710 Westwood Plaza Room 4238, Los Angeles, California 90095-1769. email: toga@ loni.ucla.edu.

This article was previously published in the Journal of Neurosurgery. 